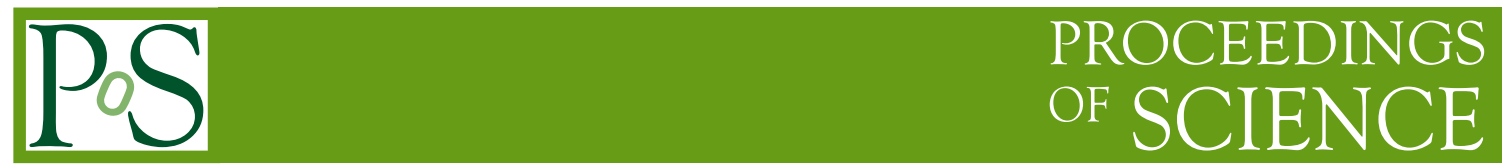

\title{
ATLAS results on quarkonia and its associated production
}

Cesare Bini*, on behalf of the ATLAS Collaboration

Sapienza Universitá di Roma and INFN Roma

E-mail: cesare.bini@romal.infn.it

Recent results from the ATLAS experiment at LHC on the inclusive production of $\mathrm{J} / \psi, \psi(2 \mathrm{~S})$ and $\Upsilon(1 \mathrm{~S}-3 \mathrm{~S})$ in $\mathrm{pp}, \mathrm{pPb}$ and $\mathrm{PbPb}$ collisions are presented and compared to theory expectations. Results on di-J/ $\psi$ production in pp collisions, including the separation of single and double parton scattering components are also discussed.

ICHEP2018, International conference on High Energy Physics

5-12 July 2018

Seoul, South Korea

${ }^{*}$ Speaker. 


\section{Introduction}

The study of quarkonia production in high-energy hadron collisions is a very effective method to test models inspired by QCD. Since the first measurements done at Tevatron [1] it was clear that models simply based on $\mathrm{pQCD}$ were not able to account for the large production cross-section. Several measurements of charmonium and bottomonium production using $\mathrm{pp}, \mathrm{pPb}$ and $\mathrm{PbPb}$ collisions at the LHC at different center of mass energies have been carried out. Charmonium production $\mathrm{PbPb}$ collisions is also a sensitive probe to study the hot, dense system created in heavy ion collisions [2] . More recently measurements of the associated production of quarkonia with vector bosons or of di-quarkonia have also been presented. In this paper the most recent results of the ATLAS experiment at LHC [3] concerning quarkonia and di-quarkonia production are presented.

Two main ingredients are required to measure quarkonia at LHC: dedicated low- $\mathrm{p}_{T}$ muon triggers able to keep the trigger rates at acceptable levels, and a method to discriminate between prompt and non-prompt charmonium production, the latter being mostly due to decays in flight of B-mesons. Both these ingredients are made possible in ATLAS and have been also successfully implemented in $\mathrm{pPb}$ and $\mathrm{PbPb}$ collision data.

\section{Quarkonia inclusive production in $\mathrm{pp}, \mathrm{pPb}$ and $\mathrm{PbPb}$ collisions}

The cross-section for inclusive production of prompt $\mathrm{J} / \psi, \psi(2 \mathrm{~S})$ and $\Upsilon(1 \mathrm{~S})$ in pp collisions at a center of mass energy of $5.02 \mathrm{TeV}$ is shown in Fig. 1 as a function of the di-muon $\mathrm{p}_{T}$ for different rapidity values and is compared to NRQCD predictions [4]. The data correspond to an integrated luminosity of $25 \mathrm{pb}^{-1}$ [5]. The charmonia data confirm the good agreement with the predictions already observed for the data at 7 and $8 \mathrm{TeV}$ center of mass energy [6]. On the other hand the $\Upsilon$ data show a clear disagreement with NRQCD predictions at low $\mathrm{p}_{T}$.
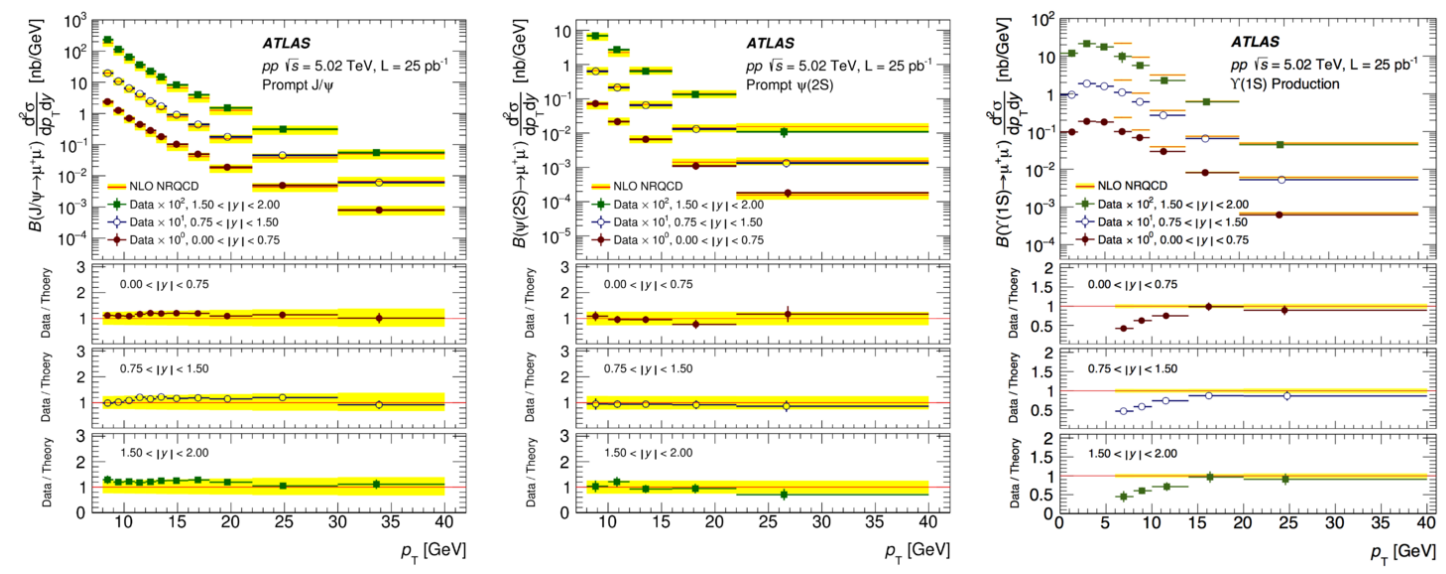

Figure 1: The differential prompt production cross section times dimuon branching fraction of $\mathrm{J} / \psi$ (left), $\psi(2 \mathrm{~S})$ (middle) and $\mathrm{\Upsilon}(1 \mathrm{~S})$ (right) as a function of transverse momentum $\mathrm{p}_{T}$ for three intervals of rapidity $\mathrm{y}$ in pp collisions at 5.02 TeV. For each increasing rapidity slice, an additional scaling factor of 10 is applied to the plotted points for visual clarity. The NRQCD theory predictions are also shown. From Ref. [5]. 

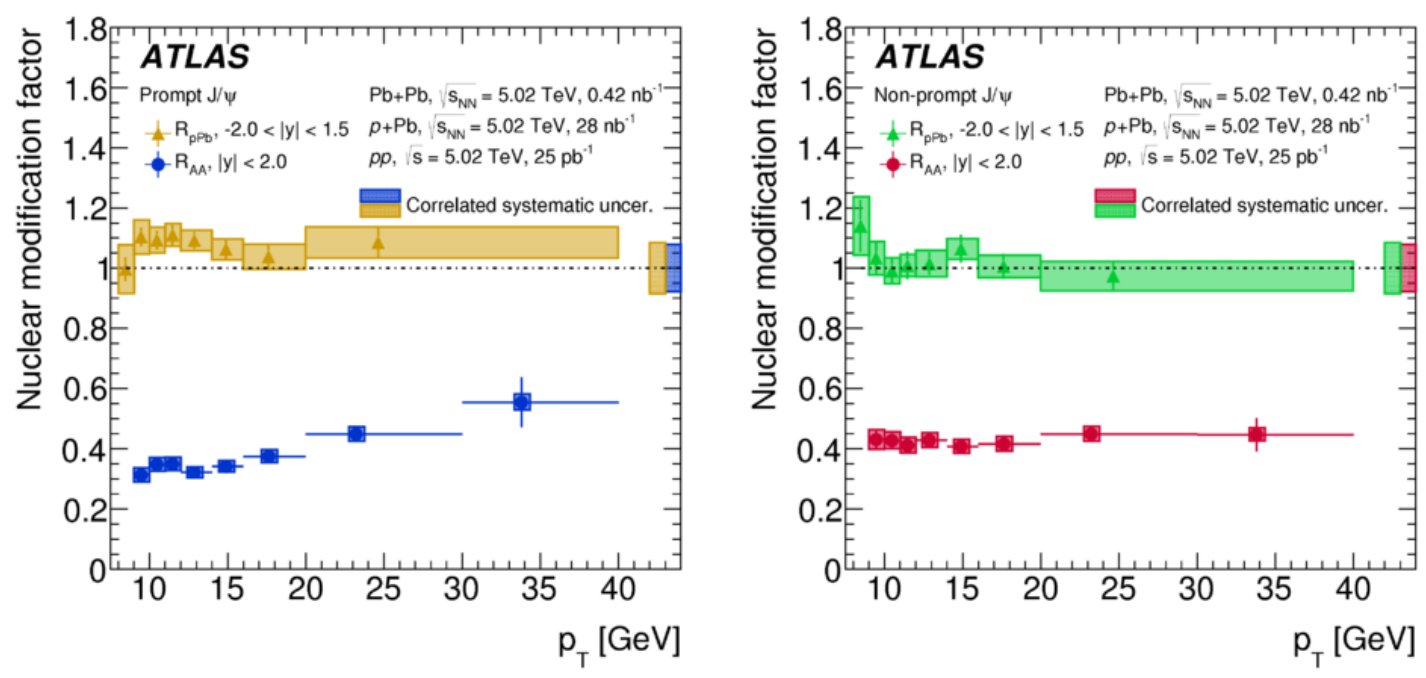

Figure 2: Comparisons of $\mathrm{R}_{A A}$ and $\mathrm{R}_{p P b}$ as a function of $\mathrm{p}_{T}$ in a similar rapidity range for the prompt (left) and non-prompt (right) $\mathrm{J} / \psi$. The statistical uncertainty of each point is indicated by a narrow error bar. The error box plotted with each point represents the uncorrelated systematic uncertainty, while the shaded error box at $y$-axis $=1$ represents correlated normalisation uncertainties. From Ref. [7].

Non-prompt charmonia production is compared to FONLL predictions [8] and shows a good agreement, better than the one observed for the corresponding 7 and $8 \mathrm{TeV}$ center of mass data.

The comparison of the pp results with $\mathrm{pPb}$ and $\mathrm{PbPb}$ at the same nucleon-nucleon center of mass energy allows to obtain the Nuclear Modification Factors $\mathrm{R}_{p P b}$ and $\mathrm{R}_{A A}$ respectively that are the ratios between the production in $\mathrm{pPb}$ and $\mathrm{PbPb}$ and the one in pp scaled to the number of effective nucleons [5]. The results for prompt $\mathrm{J} / \psi$ production as a function of $\mathrm{p}_{T}$ are shown in Fig. 2. It can be seen that no modification is found in the case of $\mathrm{pPb}$ collisions, while a large suppression is confirmed for $\mathrm{PbPb}$ collisions [7] .

\section{Di-J/ $\psi$ associated production}

The search for events with a production of two $\mathrm{J} / \psi \mathrm{s}$ has been carried on using a sample of 11.4 $\mathrm{fb}^{-1}$ at $8 \mathrm{TeV}$ center of mass energy [9]. 1170 events with two prompt $\mathrm{J} / \psi$ coming from the same primary vertex are selected. Events are classified according to their positions in the $\Delta y-\Delta \phi$ plane in order to discriminate $\mathrm{J} / \psi$ pairs coming from the same parton interaction (SPS) from $\mathrm{J} / \psi$ pairs coming from two different parton interactions (DPS). Fig. 3 shows the measured cross-section as a function of the di-J $/ \psi$ invariant mass and transverse momentum together with the estimated DPS contribution. Results are well described by QCD predictions for both contributions [10].

The fraction of DPS events with respect tot the total is: $f_{D P S}=(9.2 \pm 2.1$ (stat $) \pm 0.5$ (syst) $) \%$. From this result it is possible to estimate the effective cross-section $\sigma_{e f f}$ [11] that is: $\sigma_{e f f}=$ $(6.3 \pm 1.6$ (stat) \pm 1.0 (syst) $\pm 0.1(\mathrm{BF}) \pm 0.1$ (lumi) $) \mathrm{mb}$. This result is somehow lower than the many other estimates based on different processes [9] but on the other hand agrees with other results based on di-J/ $\psi$ measurements. 

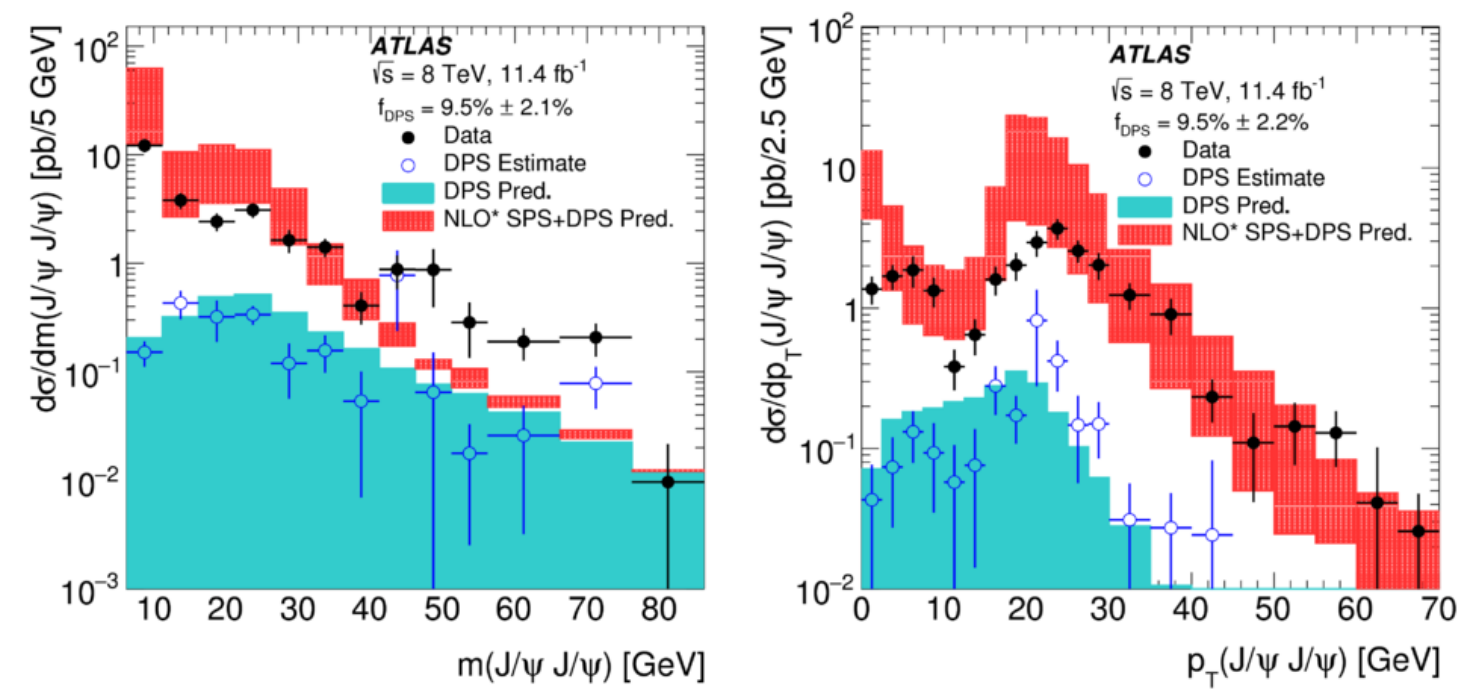

Figure 3: The estimated DPS and total differential cross-sections as a function of the invariant mass of the di-J/ $\psi$ (left) and of the transverse momentum of the di-J/ $\psi$ (right). Data are compared to the LO DPS + NLO* SPS predictions. The DPS predictions are normalised to the value of $\mathrm{f}_{D P S}$ found in the data and the NLO* SPS predictions are multiplied by a constant feed-down correction factor. From Ref. [9].

\section{References}

[1] CDF Collaboration, Phys. Rev. Lett. 79 (1997) 572.

[2] T. Matsui and H. Satz, Phys. Lett. B 178 (1986) 416.

[3] ATLAS Collaboration, JINST 3 (2008) S08003.

[4] H.Han et al., Phys.Rev. D94 (2016) 014028.

[5] ATLAS Collaboration, Eur. Phys. J. C 78 (2018) 171.

[6] ATLAS Collaboration, Eur. Phys. J. C 76 (2016) 1.

[7] ATLAS Collaboration, Eur. Phys. J. C 78 (2018) 762.

[8] M.Cacciari et al., JHEP 10 (2012) 137.

[9] ATLAS Collaboration, Eur. Phys. J. C77 (2017) 76.

[10] C. Borschensky and A. Kulesza, arXiv: 1610.00666 [hep-ph]; J. P. Lansberg and H. S. Shao, Phys.Lett.B 751 (2015) 479.

[11] B. Humpert, Phys. Lett. B 131 (1983) 461. 